\title{
0204 HEALTHCARE RESPONSE TO DOMESTIC VIOLENCE IN VIETNAM: LESSONS LEARNT FROM INTERVENTIONS AND POLICY IMPLICATIONS
}

M Le Thi Phuong, ${ }^{*}$ P Le Tuan Correspondence: UNFPA, 2E Van phuc, Ba Dinh Dist. Ha Noi City, Viet Nam, 84 -4, Vietnam

\subsection{6/ip.2010.029215.204}

This paper reviewed the lessons learnt from pilot intervention models on healthcare response to domestic violence and provided recommendations for a comprehensive healthcare strategy to support domestic violence victims in Vietnam. The intervention models were implemented at five hospitals in Vietnam. The interventions included training of health staff on gender sensitivity, domestic violence (DV), and skills to identify and support victims. In addition, guidelines for health providers working with victims were developed and used by health workers. The favourable environment for DV prevention and screen was established. The counselling centres were established in the studied hospitals to provide psychological counselling and other supports to victims.

Findings From 2002 to June 2010 more than 2300 victims of DV were identified and received medical treatment and emotional counselling. Intervention has brought about changes in health providers knowledge, attitude and practice. However, health providers have yet adequately linked their knowledge and awareness with professional duties in DV prevention and screen. Screening on sexual abuse was not culturally practical in health facilities. Follow-up with victims after discharge from hospital was limited.

Conclusions The lessons learnt from intervention models can be used for development of a comprehensive healthcare 
strategy to support GBV/DV victims. This strategy should include strengthening advocacy for health providers, especially health leaders/managers on DV prevention; ensuring health providers responsibility to addressing GBV as an essential component of their routine professional work; and monitoring the screening and providing healthcare for DV victims in health facilities. 